\title{
Case series of retained rectal foreign body. A Malaysian experience
}

\author{
Wei Soon Pang, Jih Huei Tan,Henry Chor Lip Tan, Jonathan Khee Ghee Tan \\ Pang WS, Tan JH, Tan HCL, et al. Case series of retained rectal foreign body. A Malaysian experience. Malays Fam Physician. 2021;16(2);78-82. \\ https://doi.org/10.51866/cr1087
}

\section{Keywords:}

Retained, foreign body,

rectum, Malaysia

\section{Authors:}

Tan Jih Huei
(Corresponding author)
MMed Surg. (UKM)
General Surgeon, Hospital Sultanah
Aminah, Johor Bahru, Malaysia
Email: huei_87@hotmail.com

Pang Wei Soon

MD, MRCS (Ed.)

General Surgical Trainee, Pusat

Perubatan Universiti Kebangsaan

Malaysia

\section{Henry Tan Chor Lip}

MMed Surg. (UKM)

General Surgeon, Hospital Sultanah

Aminah, Johor Bahru, Malaysia

\section{Jonathan Tan Khee Ghee}

MMed Surg. (UKM)

General Surgeon, Hospital Sultanah

Aminah, Johor Bahru, Malaysia

\begin{abstract}
Retained rectal foreign body, with its associated social stigma, is a medical condition that is infrequently reported in Malaysia. We report the surgical management of five cases of retained foreign objects in the rectum seen over a one-year period. There were three young and two elderly male patients. One of the elderly patients presented with altered bowel habits and an abdominal mass that mimicked a symptomatic colonic tumor. All patients had an abdominal radiograph which clinched the diagnosis of a retained foreign rectal body. Successfully retrieved objects included two bottles, one bidet device, and two sex toys. Open surgery for retrieval of the impacted rectal foreign body was required in one patient. All underwent successful retrievals without any adverse postoperative complications. In our case series, retained rectal foreign bodies were observed only in the male gender. The age presentation was bimodal, with age groups in the twenties and sixties. The treatment options used for the retrieval of these impacted foreign bodies included transanal extraction or explorative laparotomy. Proximal migration and delayed presentation are possible indications for the latter approach.
\end{abstract}

\section{Introduction}

Retained foreign bodies (FBs) in the rectum have been reported since the 16th century. The majority were retrospective case series from western populations. ${ }^{1}$ However, the report by Odagiri et al. had the largest number, with 684 retained rectal FBs from the Japanese population. $^{2}$ In the conservative Asian population, such as in Malaysia, published data for retained rectal FBs were limited, probably due to underreporting resulting from the attached social stigma. ${ }^{3}$ After an extensive literature search, only two reports from the local Malaysian population were found over a period of two decades. They described five patients with rectal FB insertion for sexual gratification purposes. ${ }^{3,4}$

The patients often presented late, when symptoms became intolerable, due to the embarrassing nature of the condition and cultural stigma involved. ${ }^{1,3}$ FBs are often inserted into the rectum for sexual pleasure or habitual usage associated with abnormal psychosocial conditions. Patients often present after exhausting all efforts in self-removal. We discuss five patients with retained FBs in the rectum that were treated by the general surgery team from 2018-2019. The clinical presentation and treatment strategies for safe retrieval were narrated in this case series.

\section{Case 1}

A 25-year-old man presented with complaint of acute lower abdominal pain. On further history-taking, he admitted to the insertion of a vibration device through his anus for sexual pleasures. In this incident, the device migrated too deeply into his rectum resulting in failed self-retrieval. He tried to retrieve the device by digital manipulation and bearing down but was unsuccessful after repeated attempts for one day.

Social history revealed the patient was single, and no history of psychiatric illnesses was elicited. Physical examination revealed that the patient was generally well with normal blood pressure and no tachycardia. Abdominal palpation revealed a soft, mildly distended abdomen with no signs of peritonism. There were no masses palpable in the abdomen. Upon digital rectal examination, a hard edge of an object was felt at about $8 \mathrm{~cm}$ from the anal verge. Proctoscopic examination under spinal anesthesia revealed an $8 \mathrm{~cm}$-long plastic vibrator. Successful removal was performed transanally with suprapubic pressure and per anal manipulation (Figure 1). An anal tear at the 12 o'clock position was identified, which was consistent with a history of digital manipulation. An immediate sigmoidoscopy following retrieval revealed mucosal erosions 
at the mid rectum without evidence of rectal perforation. He was discharged well but refused follow up.

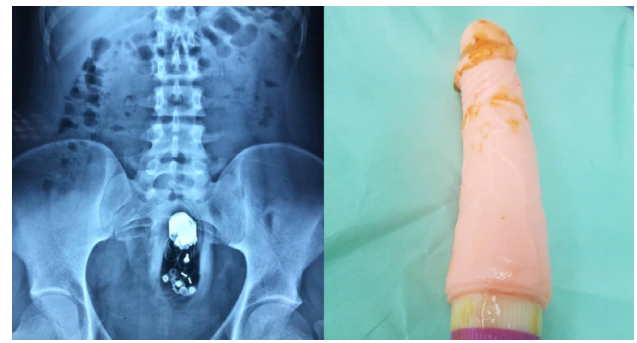

Figure 1: Supine abdominal radiograph and vibrator device extracted from Case 1.

\section{Case 2}

A 29-year-old man presented with anal pain and bloody per-rectal discharge of one-day duration. Physical examination revealed an abnormal hose tailing out of the anal orifice. The patient revealed that there was a bidet device lodged within his rectum. Initial history was conflicting with a suggestion of accidental insertion of a FB. After further questioning, the patient admitted he had a history of habitual bidet insertion into his anus for sexual gratification. Social history revealed that the patient was married with children but had no prior psychiatric illnesses. The patient was clinically well and hemodynamically stable. Upon examination, the abdomen was soft, no masses were palpable, and there was no evidence of peritonism. Per-rectal examination showed a plastic hose dangling out from his anus attached to a bidet device. The patient was managed in a similar manner to Case 1. He underwent examination and retrieval under regional anesthesia. The bidet was manually retrieved successfully without significant injuries to the bowel (Figure 2). A sigmoidoscopic assessment immediately following the removal revealed superficial mucosal lacerations at the anal canal and lower rectum. The patient was then discharged well but he refused follow up.
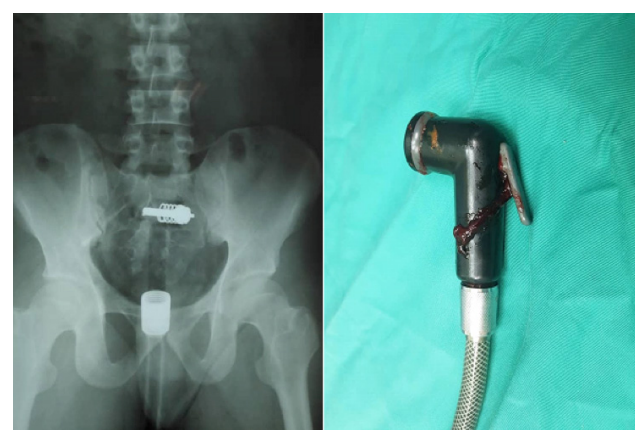

Figure 2: Supine abdominal radiograph and bidet extracted from Case 2 .

\section{Case 3}

A 73-year-old man without comorbidities presented with a history of abdominal discomfort, altered bowel habits, and a left-sided abdominal swelling of one week duration. The swelling had subsequently migrated to the suprapubic region. The patient vehemently denied any history of ingesting or inserting any FBs into himself. He lived with his wife and family and denied any history of psychiatric illnesses. The patient was clinically well with no tachycardia or hemodynamic compromise. Upon examination, the abdomen was soft, not distended, and a hard irregular tubular mass was felt over the lower abdomen. There was no palpable mass on digital rectal examination. An abdominal radiograph revealed a radiolucent cylindrical FB possibly located in the sigmoid or descending colon. Despite the conflicting history, the abdominal radiograph shown (Figure 3) clearly clinched the diagnosis of an intra-abdominal FB. The patient then underwent a midline laparotomy and the surgeons found a hard cylindrical mass within the descending colon. The FB (Figure 3) was retrieved via a colotomy of the involved colonic segment. There was evidence of colitis due to thickening of the colonic wall. The colotomy incision was repaired primarily. Recovery was uneventful and the patient was discharged on postoperative day three. Similarly, the patient refused further surgical follow-up treatment.

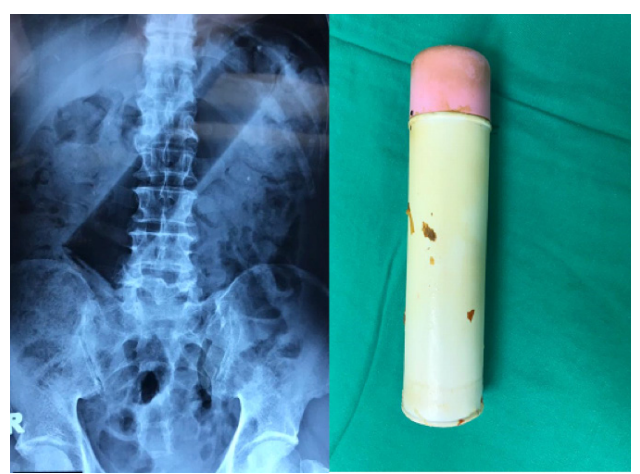

Figure 3: Supine abdominal radiograph and talcum powder container extracted from Case 3.

\section{Case 4}

A 68-year-old man with diabetes and hypertension confessed to inserting a hairspray canister into his anus on the morning of the day of his presentation. He had constipation for the past 2 months and used the hairspray canister daily to stimulate bowel opening. As soon as the canister slipped beyond the anal verge, it became irretrievable. Social history revealed that the patient was married and 
did not have any past psychiatric illnesses. He was well and hemodynamically stable. Upon examination, his abdomen was soft and no mass was palpable. Digital rectal examination did not reveal any particular abnormalities. He then underwent rectal examination under general anesthesia. The hairspray canister was manually retrieved transanally with the help of external pressure over the lower abdomen. Limited colonoscopy into the descending colon revealed no evidence of bowel perforation. There were minimal mucosal lacerations at the sigmoid colon and upper rectum. The bowel was circumferentially erythematous, with sloughy and thickened mucosa due to FB irritation. A subsequent psychiatric evaluation found no major mental illnesses. Postoperative recovery was uneventful, and he was subsequently discharged after 24 hours of observation. Similar to the other patients, he refused subsequent treatment follow up.

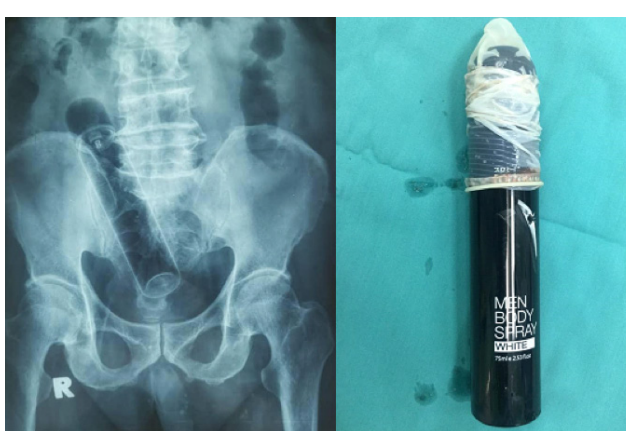

Figure 4: Supine abdominal radiograph and hair spray container extracted from Case 4.

\section{Case 5}

A 38-year-old man presented with history of self-insertion of a sex vibrator into his anus a few hours prior to being seen. Multiple attempts at self-removal failed due to proximal migration of the FB. He claimed that the electrical cord attached to the vibrator had snapped as he attempted to remove it. He was not married and had no known psychiatric illness. Upon examination, his abdomen was soft with no palpable masses. There was also no mass noted on digital rectal examination. An urgent colonoscopy revealed that the vibrator was impacted at the rectosigmoid colon. The retrieval procedure was performed under monitored sedation in the endoscopy suite. The FB was retrieved with a combination of endoscopic snare device and digital manipulation. Following the removal of the $\mathrm{FB}$, he was fit for discharge but refused a scheduled follow up.

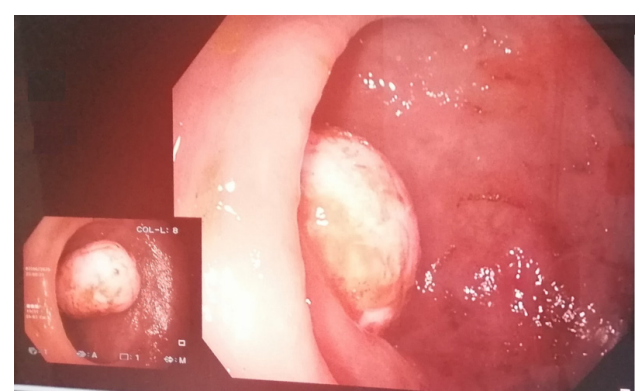

Figure 5: Endoscopic view of the sex toy at rectosigmoid colon for Case 5 . The hidden end on another side was attached by the broken electrical wire.

\section{Discussion}

The rectum is a naturally collapsible anatomical structure armed with reflexes allowing for storage or defecation of feces. Furthermore, the rectum produces mucus that acts as a lubricant, allowing retained foreign objects to migrate proximally. Typically, most patients would attempt manual self-removal and seek medical attention after multiple futile attempts. This often results in mucosal and anal injury. The mean time of presentation after insertion in one study was 23 hours, with a range of 6-72 hours. ${ }^{5}$ In Case 3 of our series, there was a delay in presentation for more than a week, suggesting that elderly patients may be more tolerant of a retained FB. Previous case series have observed a bimodal age trend for retained FB. Our group of patients revealed the same bimodal pattern-three young and two elderly patients.

Rectal FBs are either inserted voluntarily or involuntarily. Voluntarily inserted rectal FBs may be for sexual or non-sexual purposes. The most common non-sexual purpose is for rectal stimulation to alleviate constipation. ${ }^{1}$ An example of this is Case 4 of our series. Three cases admitted to inserting the rectal FBs for sexual gratification. The FBs found in the rectum were not limited to sex toys such as those seen in Cases 1 and 5. Common objects found in other reported series include common household objects such as bottles and glasses. ${ }^{1}$ These objects were seen in Cases 3 and 4 in our series. Other reported objects were cucumbers, carrots, wood, rubber objects, light bulbs, fluorescent light tubes, axe handles, broomsticks, utensils, and decorative ornaments. These objects have irregular surfaces with attached flanges or cords that could prevent them from being readily retrieved from the rectum. ${ }^{1}$ In Case 2, the man had habitual insertion of a bidet device for sexual gratification. To date, we are not aware 
of any published case reports in the English literature depicting the impaction of a bidet in rectum.

In general, the patient's evaluation begins with an accurate history-taking to identify the cause of the FB insertion and the nature of the impacted object. This should be performed in a professional and non-judgmental manner in order to not upset the patient. On physical examination, the FB may not be felt on digital rectal examination as it may have migrated proximally into the sigmoid colon. Occasionally, it may be palpable on abdominal examination mimicking a tumor, such as in Case 3. An abdominal radiograph is an important diagnostic modality to clinch the diagnosis of a retained $\mathrm{FB}$ unless the object is radiolucent in nature. ${ }^{7}$ In addition, an abdominal CT scan is useful to provide information on associated complications of rectal and colonic perforations. ${ }^{8}$

Outpatient bedside extraction in the emergency room has a success rate of $75 \%{ }^{1}$ Failure of bedside retrieval warrants an inpatient extraction under sedation, regional, or general anesthesia. The object can be extracted transanally under direct vision, endoscopically, or by open surgery. The methods used to extract the retained rectal FBs vary with the type and nature of the FBs. The preferred method of extraction depends on the size and shape of the object (sharp vs. blunt, smooth vs. rough), the site (distance from anal verge), and the presence of bowel perforation or peritonism. For proximally located FBs, it is safe to extract the object via an endoscopic approach. ${ }^{1}$ However, in Case 3, endoscopic retrieval was not attempted for safety reasons. First, the large dimensions of the object increased the index of difficulty for endoscopic retrieval. Second, the object may have migrated too proximally in the bowel, which made endoscopic retrieval technically difficult. Third, the delayed presentation may be associated with distal bowel congestion, edema, and inflammation, which increase the risk of bowel perforation. With regards to sharp or oddly shaped objects, a surgical approach (laparotomy and FB removal via enterotomy) is the preferred choice. Transanal extraction is the preferred method for lowlying rectal FBs with smooth edges and short duration of entrapment. ${ }^{5}$
After successful retrieval of a retained rectal $\mathrm{FB}$, an endoscopic assessment is mandatory to assess the involved bowel for mucosal injuries. ${ }^{1}$ Subsequent appropriate psychiatric evaluation, advice, and counseling should be offered to these patients to prevent future recurrences. Unfortunately, four of our patients rejected psychiatric assessment. The only patient (Case 4) evaluated by the psychiatric team had no major mental disorder. All the patients refused follow up, probably due to embarrassment. Prior to discharge, all patients were given ample advice on signs of possible delayed bowel perforation and offered early surgical follow up.

Our present cohort of patients did not report any causes of retained rectal FB related to abuse or sexual assault. However, the occurrence of such violent acts is a less common cause of rectal FBs. A review from Kyle et al. proposed a well-designed algorithm for treatment of rectal FBs. The management encompasses initial evaluation, extraction technique (transanal, endoscopic, and operative), and post-extraction care. Factors that may influence the choice of rectal FB extraction technique include presence of peritonitis, evaluation by digital rectal examination, and endoscopic assessment. ${ }^{1}$

\section{Conclusion}

The incidence of retained rectal FBs in Malaysia may be low due to underreporting. In the majority of cases, these objects can be safely extracted via the transanal approach. Deeply migrated objects in the elderly may be misdiagnosed as obstructed rectal tumors. Abdominal radiography is necessary to ascertain the diagnosis, especially in patients with possible fabricated or concealed history of FB insertion. Proximally migrated FBs that cannot be retrieved by transanal or endoscopic extraction warrant an explorative laparotomy. Physical evidence of peritonitis warrants an urgent laparotomy and precludes endoscopic retrieval. Open surgery is also considered when there is anticipation of bowel perforation and injury due to prolonged retention of the FB. Early diagnosis of retained rectal FBs is likely to result in less invasive or non-operative management. 


\section{How does this paper make a difference to general practice?}

- The incidence of retained rectal foreign body is increasing within the local Malaysian population. Patients with constipation may present with a history of habitual transanal insertion of a foreign body.

- The majority of such sufferers often provide illogical and vague history regarding this embarrassing clinical condition.

- An abdominal radiograph has a high specificity to clinch the diagnosis.

- Referral to a center with general surgical service is warranted in the retrieval of impacted rectal foreign objects.

\section{References}

1. Cologne KG, Ault GT. Rectal Foreign Bodies: What Is the Current Standard? Clin Colon Rectal Surg. 2012 Dec;25(4):214-8.

2. Odagiri $H$, Yasunaga $H$, Matsui $H$, Fushimi $\mathrm{K}$, lizuka T, Kaise M. Difference in Outcomes of Rectal Foreign Bodies between Males and Females: A Retrospective Analysis of a National Inpatient Database in Japan. Digestion. 2015;92(3):165-70.

3. Chor Lip, H.T., Huei, T.J. and Tuan Mat, T.N.A., 2017. Removal of an impacted rectal foreign body - avoiding laparotomy. Sri Lanka Journal of Surgery, 35(3), 25-26.
4. Sangar P, Henry G, Sood S. Foreign bodies in the rectum: report of a case series and review of the literature. Sri Lanka Journal of Surgery. 2013 Aug 18;31(2):27-30.

5. Coskun A, Erkan N, Yakan S, Yildirim M, Cengiz F. Management of rectal foreign bodies. World J Emerg Surg. 2013 Mar 13;8(1):11.

6. Kokemohr P, Haeder L, Frömling FJ, Landwehr P, Jähne J. Surgical management of rectal foreign bodies: a 10-year single-center experience. Innov Surg Sci. 2017 Jun;2(2):8995.
7. Kit Fai Lee. John Wong, Paul BS Lai. Radiolucent foreign body visible on plain radiography Can J Surg. 2008 Aug; 51(4): E87-E88

8. Emi Sanjo, Fumihiko Tamamoto, Shoichi Ogawa, Maiko Sano, Tetsunori Yoshimura, Miwako Nozaki, "Unexpected Radiologic Findings for a Casting Type of Radiolucent Colorectal Foreign Body Composed of Polyurethane Foam", Case Reports in Radiology, 2016, 4987105. 